\title{
A POSSIBLE NOVA FROM THE IRAS POINT SOURCE CATALOGUE
}

P. Garcia-Lario(1), A. Manchado(1), K.C. Sahu(2), S.R. Pottasch(2)

(1) Instituto de Astrofisica de Canarias, E-38200, La Laguna, Tenerife, Spain

(2) Kapteyn Laboratorium, NL-9700 AV Groningen, The Netherlands

ABSTRACT. During a spectroscopic survey of IRAS sources with infrared colours similar to those in planetary nebulae, IRAS 06518-1041 revealed itself as a possible novalike object. The spectrum of IRAS 06518 - 1041, which is shown in Figure 1, presents strong and broad hydrogen Balmer lines together with broad permitted lines of He and C. This spectrum also shows a much weaker narrow nebular component, with forbidden lines of [OIII] and narrow $\mathrm{H}_{\alpha}$. The mean velocity associated to the observed line widths is about $800 \mathrm{~km} \mathrm{~s}^{-1}$. The observed emission lines correspond well to typical post-novae objects in the quiescent phase (Warner, 1989). Another argument which supports the idea of this object being a nova-like star is that there are some indications of the binarity of the source if we attend to the asymmetries observed both in the $\mathrm{H}_{\beta}$ and $\mathrm{H}_{\alpha}$ profiles. The high resolution spectrum at $\mathrm{H}_{\alpha}$ shows the presence of a sharp double-peaked central emission from a low expanding envelope at a velocity of $35 \pm 5 \mathrm{~km} \mathrm{~s}^{-1}$, together with a much broader emission with velocities of several hundred kilometers. The displacement between both components is about $130 \mathrm{~km} \mathrm{~s}^{-1}$. This broad component could be due to the presence of an accretion disk around the system with hydrogen-rich material falling onto the compact companion. This is supported by the fact that the broad emission looks like the combination of different velocity components giving rise to a complex and asymmetric line profile. From this high resolution spectrum we derive a $\mathrm{V}_{\boldsymbol{L S R}}=57$ $\mathrm{km} \mathrm{s}^{-1}$, which points to a distance of $5.5 \mathrm{kpc}$ if we adopt the model rotation curve of the Galaxy given by Pottasch (1984) and a distance of $9 \mathrm{kpc}$ from the Sun to the galactic center. The large infrared excess detected by the IRAS satellite is probably the result of the formation of dust grains in the outer parts of the common envelope at a temperature of about $160 \mathrm{~K}$, as can be derived from the infrared data. This infrared excess is also present in other nova-like objects and is specially important in old and slow novae (Callus et al, 1987) where the continuous ejection of mass produces dust shells which are strong emitters in the infrared.

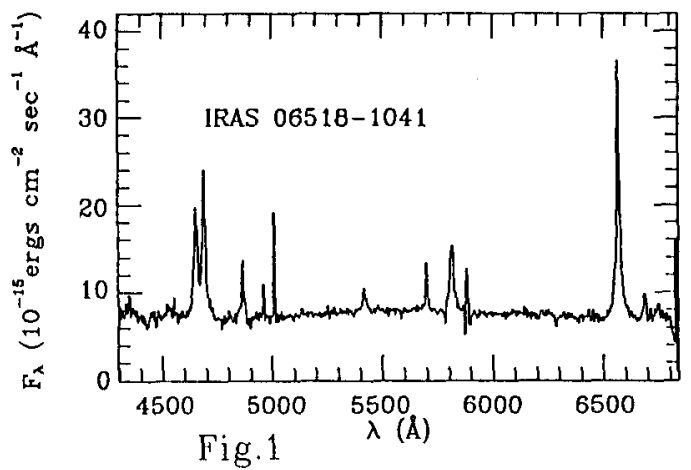


We must note here that the broad emission lines of $\mathrm{He}, \mathrm{N}$ and $\mathrm{C}$ are also common features in Wolf-Rayet stars, which may give an alternative interpretation to the spectra, but Wolf-Rayet are characterized by their extreme hydrogen deficiency. No hydrogen lines at all, or only traces of them, are found in their spectra. Sometimes WR nuclei of planetary nebulae can produce similar features, even novae-like outbursts, like in the case of A58 (or V605 Aql) which was initially classified as a peculiar nova, this outburst being possibly connected to the He flash of the central star (Duerbeck, 1987). Whatever it is, a post-nova candidate, a single Wolf-Rayet star with high hydrogen abundance or a close binary sistem (WR + compact companion) it is clear that it is a peculiar object which should be monitored at different ranges of wavelength in order to determine its real nature and if its possible connection with the novae phenomena is true or not.

\section{References}

Duerbeck, H.W.: 1987, Sp. Sc. Rev., 45, 1/2

Pottasch, S.R.: 1984, Planetary Nebulae, ed. Reidel-Dordrecht

Warner, B.: 1989, in Classical Novae, eds. M.F. Bode and A. Evans, p. 1 